\title{
Photocatalytic Performance of the MOF-Coating Layer on SPR- Excited Ag Nanowires
}

\author{
Xi Chen,* Yanshuang Zhang, Xiangyun Kong, Kun Yao,* Lingzhi Liu, Jiali Zhang, Zanru Guo, \\ Wenyuan Xu, Zhili Fang, and Yongxin Liu*
}

Cite This: ACS Omega 2021, 6, 2882-2889

Read Online

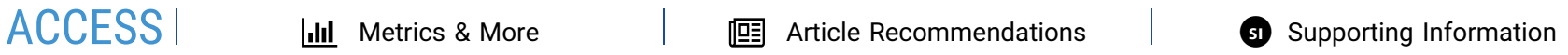
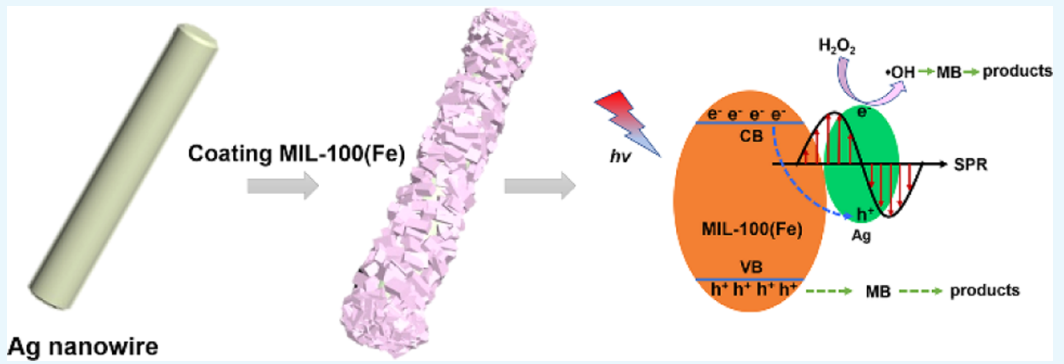

ABSTRACT: The photoactive metal-organic frameworks (MOFs) were controllably coated on the surface plasmon resonanceexcited $\mathrm{Ag}$ nanowires in a layer manner to adjust the photocatalytic activity. The influence of the thickness of the MOF coating layer on the photocatalytic activity was investigated. A thicker MOF coating layer not only facilitated the photogenerated electron-hole separation efficiency but also provided a larger Brunauer-Emmett-Teller surface area, thus enhancing the photocatalytic activity. This work provided a new way to adjust the photocatalytic activity of the photoactive MOF.

\section{INTRODUCTION}

Wastewater from dyeing, papermaking, leather, plastics, and medicine industries contains a large number of organic dyes which are difficult to be biodegraded, seriously endangering the health of aquatic animals and plants as well as human beings. ${ }^{1-4}$ The photocatalytic degradation of organic dyes in wastewater by using solar energy is green and less expensive, being considered to be one effective method for water pollution treatment. ${ }^{5-7}$ The $\mathrm{TiO}_{2}$ photocatalytic system is the first catalyst found to be used for photocatalytic degradation of organic pollutants. ${ }^{8}$ Since then, a variety of inorganic semiconductor materials including metal oxides (such as $\mathrm{ZnO}$ and $\left.\mathrm{WO}_{3}\right)^{9,10}$ and metal sulfides (such as $\mathrm{CdS}$ and $\mathrm{ZnS})^{11,12}$ have been developed and applied to photocatalytic degradation of organic pollutants. However, most inorganic semiconductor materials are with no porosity, low specific surface area, and poor adsorption of organic dye pollutants, which hinder further improvement of photocatalytic efficiency.

Metal-organic frameworks (MOFs) with a large specific surface area, adjustable crystal structure, and adjustable pore size are a new kind of porous crystal materials developed rapidly in recent years, having been applied to gas adsorption, ${ }^{13-15}$ separation, ${ }^{16-18}$ catalysis, ${ }^{19-21}$ delivery, ${ }^{22-24}$ sensors, ${ }^{25-27}$ and other fields. Under light conditions, in some MOFs, the photogenerated electrons and holes are excited to the lowest unoccupied molecular orbital and the highest occupied molecular orbital, respectively, completing the separation of photogenerated electrons and holes, after which the electrons and holes can undergo photocatalytic reactions. ${ }^{28}$ These MOFs have been used in photocatalytic degradation of organic dyes, photocatalytic reduction of metal ions, synthesis of organic compounds, hydrogen, or oxygen production from pyrolysis water, $\mathrm{CO}_{2}$ reduction, and other fields. For photodegradation of organic dyes, there are MOFs with $4,4^{\prime}$ diimidazole biphenyl and trimesic acid as the main organic ligands and $\mathrm{Mn}^{2+}$ and $\mathrm{Co}^{2+}$ as the main metal centers, ${ }^{29}$ UTSA-38, ${ }^{30}$ MOF-5, ${ }^{31} \quad \mathrm{NH}_{2}-\mathrm{UiO}-66,{ }^{32} \mathrm{NH}_{2}$-MIL-88B(Fe), MIL-100(Fe), ${ }^{33}$ ZIF-8, ${ }^{34}$ and so forth.

Like other single-component photocatalysts, MOFs also undergo a rapid electron-hole recombination process after being stimulated by light, resulting in a significant reduction in catalytic efficiency. To solve this problem, other species including noble metals and semiconductors are usually introduced into MOFs as the co-catalysts to promote the generation and separation of photogenerated electrons and holes. $^{35-39}$ At present, studies have shown that the photo-

Received: October 27, 2020

Accepted: January 8, 2021

Published: January 19, 2021 


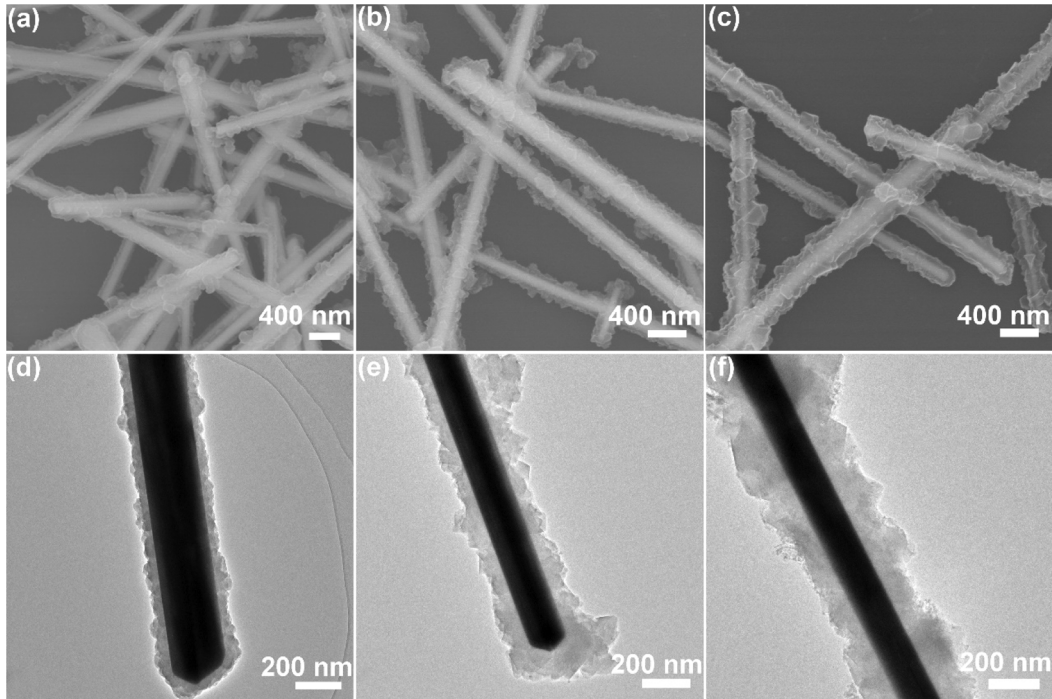

Figure 1. Scanning electron microscopy (SEM) (a-c) and TEM imaging (d-f) of MOF@Ag-2, MOF@Ag-4, and MOF@Ag-6, respectively.
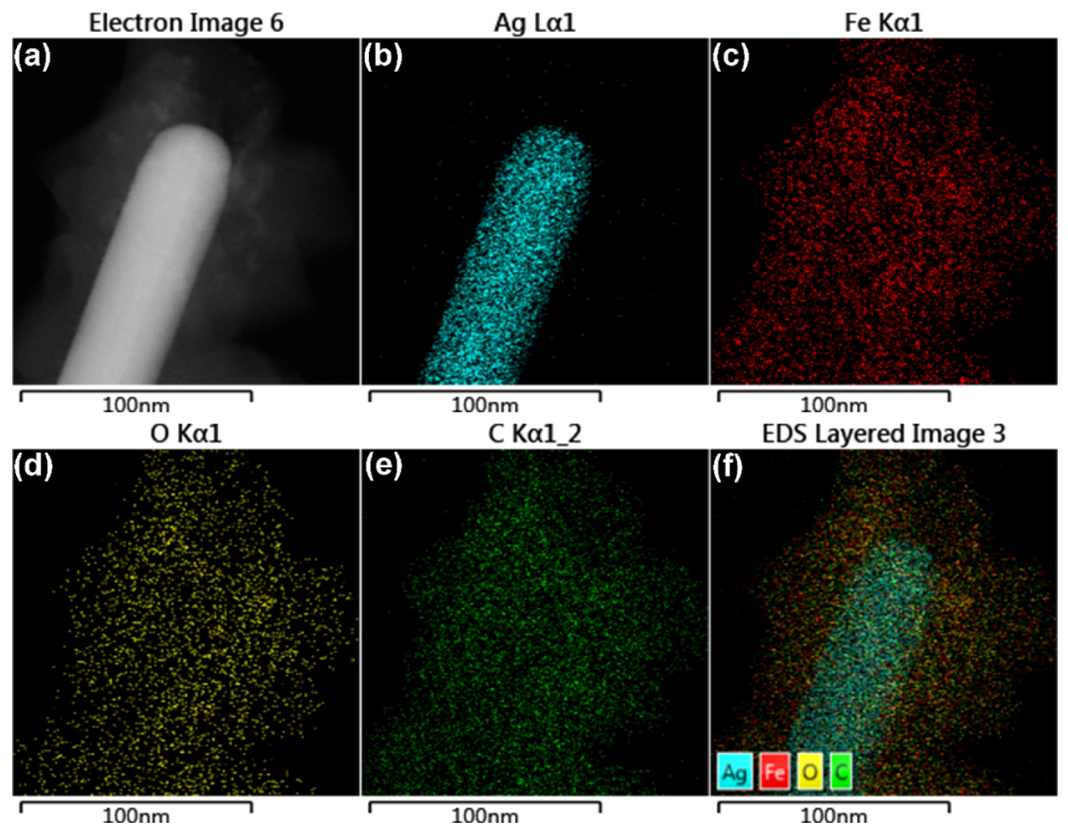

EDS Layered Image 3

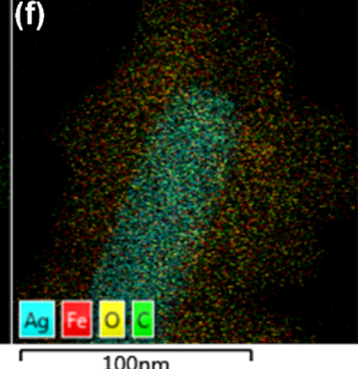

Figure 2. Electron image (a), elemental mapping images (b-e), and EDS layered image (f) of MOF@Ag-6.

catalytic effect of noble metals such as gold $(\mathrm{Au})$ and platinum (Pt) combined with MOFs is good. When conducting electrons, the photocatalytic performance of the system is improved by local surface plasmon resonance (SPR) of these noble metals. ${ }^{40-43}$ Compared with the high cost of $\mathrm{Au}$ or $\mathrm{Pt}$, silver (Ag) with high conductivity and thermal conductivity has a relatively low cost. ${ }^{44}$ So far, there are various Ag-modified MOFs that show good photocatalytic degradation of organic dyes. $^{45-47}$ However, the Ag used to modify MOFs in these reports is almost spherical nanoparticles, while $\mathrm{Ag}$ nanowires are rarely used. Unlike $\mathrm{Ag}$ nanoparticles that only possess a narrow SPR absorption at around $400 \mathrm{~nm}$, anisotropic $\mathrm{Ag}$ nanowires are known to exhibit a strong and broad SPR absorption feature in the wavelength range of 330-900 nm, ${ }^{48}$ making them become ideal nanomaterials for enhancing the photocatalytic performance of MOFs under solar light irradiation. Therefore, controllably combining Ag nanowires and MOFs, for example, Ag nanowires are homogenously coated with MOF layers, and exploring the synergistic effect between them in the photocatalytic degradation of organic dyes is interesting and promising.

Here, MIL-100(Fe) was selected to coat homogenously on $\mathrm{Ag}$ nanowires to photocatalyze the degradation of methylene blue (MB), a common cationic dye. MIL-100(Fe) is one kind of Fe-based MOF assembled by the coordination between $\mathrm{Fe}^{3+}$ ions and trimesic acid. ${ }^{49}$ The framework of MIL-100(Fe) has two kinds of pores with the sizes of 2.5 and $2.9 \mathrm{~nm}$, respectively, and the pore wall is full of benzene rings, both of which facilitate the adsorption of organic dyes. MIL-100(Fe) is an environmental-friendly MOF with high structural stability and biocompatibility in aqueous solution. In addition, MIL$100(\mathrm{Fe})$ has semiconductor properties and is an excellent photocatalytic material with unique advantages in the wastewater purification field. ${ }^{50,51}$ In our previous work, MIL$100(\mathrm{Fe})$ was controllably and homogenously coated on the surface of $\mathrm{Fe}_{3} \mathrm{O}_{4}$ and the $\mathrm{MB}$ adsorption test presented that an 

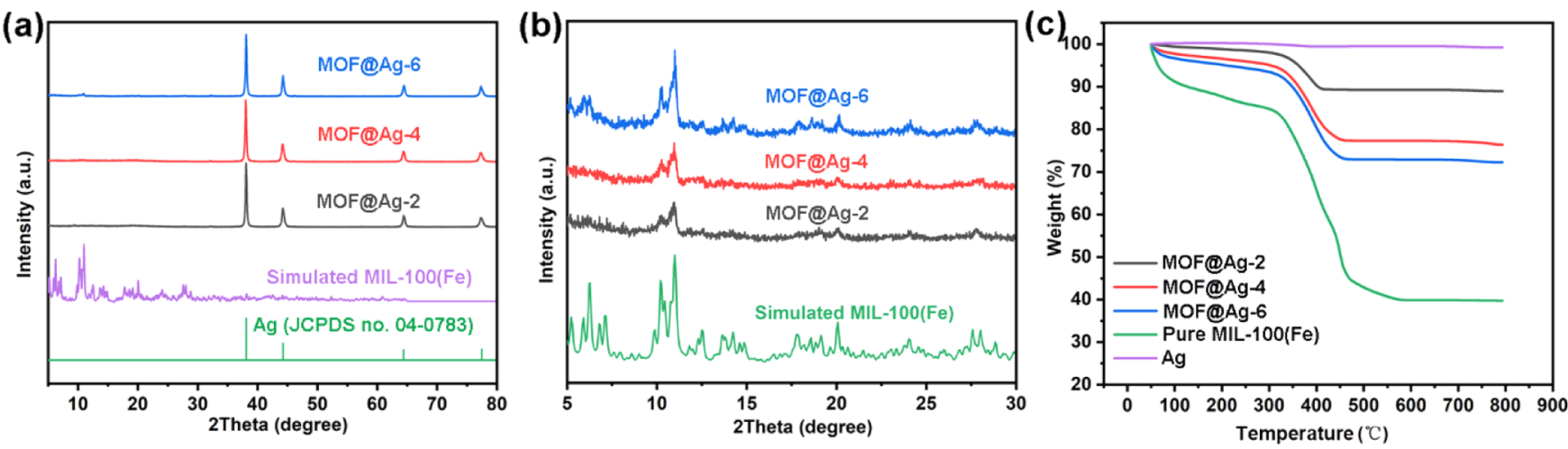

Figure 3. XRD patterns (a,b) and TG curves (c) of MOF@Ag-2, MOF@Ag-4, and MOF@Ag-6, respectively.
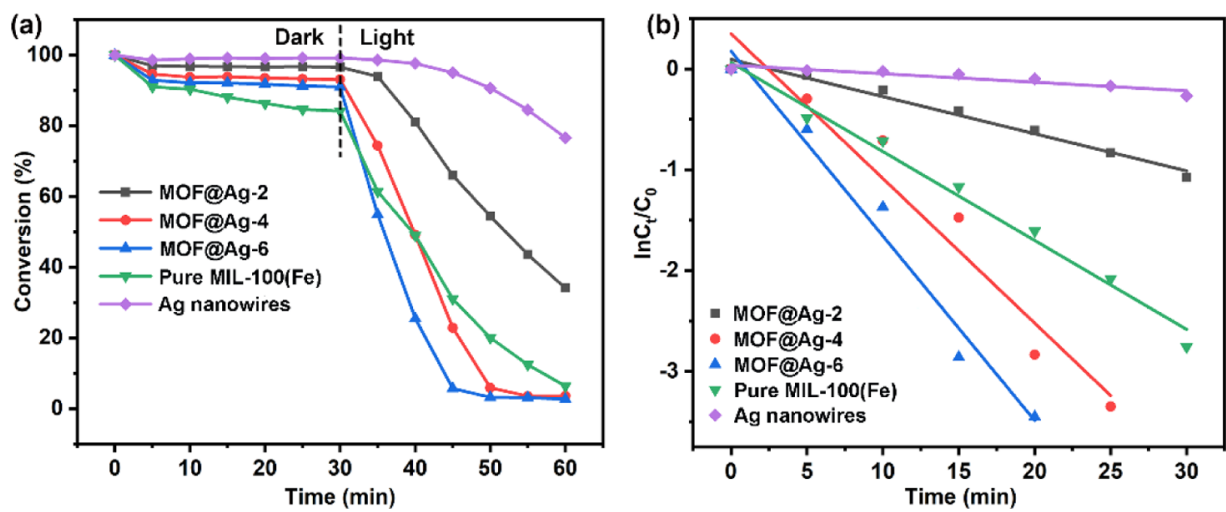

Figure 4. Photocatalytic degradation of MB (a) and corresponding kinetics study (b) over the pure Ag nanowires, MOF, MOF@Ag-2, MOF@Ag4, and MOF@Ag-6, respectively.

optimized adsorption capacity and adsorption efficiency could be achieved through enhancing the thickness of the MIL$100(\mathrm{Fe})$ layer. $^{52}$ Hence, MIL-100 $(\mathrm{Fe})$ is a desirable layer material to homogeneously coat on $\mathrm{Ag}$ nanowires to explore the photocatalytic performance of MOF-Ag composite nanowires on the degradation of organic dyes. Besides, a series photoelectrochemical tests including ultraviolet-visible diffuse reflectance spectroscopy (UV-vis DRS) analyses, photoluminescence test, and photocurrent measurement were carried out to study the synergistic effect between the MOF coating layer and Ag nanowire as well as the influences of the MOF coating layer thickness on the photocatalytic performance.

\section{RESULTS AND DISCUSSION}

Three samples of Ag nanowires with 2 cycle, 4 cycle, and 6 cycle MOF coating layers, named as MOF@Ag-2, MOF@Ag4, and MOF@Ag-6, respectively, were prepared here to investigate the morphologies and properties. Before the coating MOF layer, the surface of $\mathrm{Ag}$ nanowires is smooth (Figure S1). After coating the MOF layer, the surface of $\mathrm{Ag}$ nanowires became coarse due to the formation of MOF crystals, wrapping tightly around $\mathrm{Ag}$ nanowires (Figure $1 \mathrm{a}-\mathrm{c}$ ). As the cycles of MOF coating increases, the surface of the nanowires becomes rougher because the wrapped MOF crystals grew bigger and the thickness of the MOF coating layer got thicker. The thickness of the MOF coating layer could be observed obviously under the observation of transmission electron microscopy (TEM). The morphology of one random nanowire of each sample suggested that the thicknesses of the MOF coating layer were about 48, 86, and 122 nm for MOF@Ag-2, MOF@Ag-4, and MOF@Ag-6, respectively (Figure 1d-f).

To further identify the successful coating of the MOF layer on Ag nanowires, the electron image, elemental mapping images, and energy-dispersive X-ray spectroscopy (EDS)layered image of MOF@Ag-6 were studied (Figure 2). The electron image of MOF@Ag-6 presented obvious Ag nanowires and relatively faint MOF coating layers due to the presence of organic ingredients in the MOF (Figure 2a). The distribution of $\mathrm{Ag}$ elements was well-bounded showing an obvious nanowire morphology (Figure $2 \mathrm{~b}$ ). The distributions of $\mathrm{Fe}, \mathrm{O}$, and $\mathrm{C}$ elements together painted the MOF coating layer, as shown in Figure $2 c-e$; on account of this, we infer that the MOF was made up of $\mathrm{Fe}, \mathrm{O}$, and $\mathrm{C}$ elements. Finally, an EDS layered image of MOF@Ag-6 showed the position relationship among the elements, further identifying that the MOF coating layer was all around the Ag nanowires.

The crystal properties of MOF@Ag-2, MOF@Ag-4, and MOF@Ag-6 were investigated by powder X-ray diffraction (PXRD) patterns. As illustrated in Figure 3a, the diffraction peaks corresponding to standard Ag (JCPDS no. 04-0783) are so strong that the diffraction peaks associated with simulated MIL-100(Fe) are almost invisible. However, the diffraction peaks associated with simulated MIL-100(Fe) emerge after removing the background action of the Ag nanowire characteristic peaks, as illustrated in the XRD spectrum with a $2 \theta$ range of $5-30^{\circ}$ (Figure $3 \mathrm{~b}$ ). MOF@Ag-2, MOF@Ag-4, and MOF@Ag-6 presented the Fourier-transform infrared (FT-IR) properties of pure MIL-100(Fe), and the detailed description of FT-IR curves is presented in the Supporting Information (Figure S2a). From the thermogravimetric (TG) 

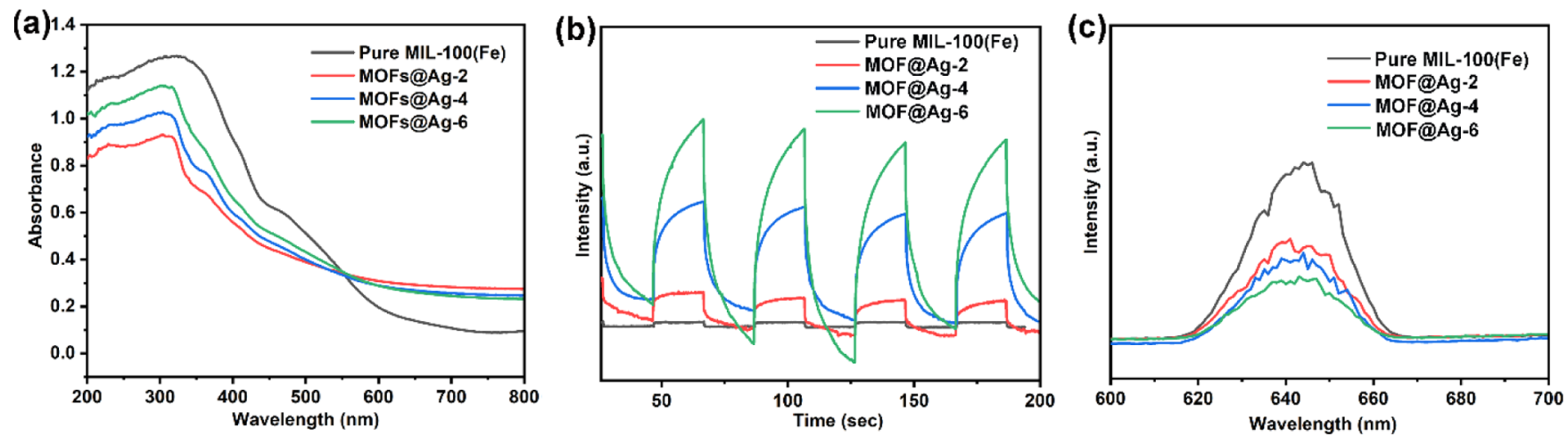

Figure 5. UV-vis DRS spectra (a), transient photocurrent response (b), and PL spectra (c) of the catalysts.

analyses (Figure 3c), there are two obvious weight loss steps in the samples of MOF@Ag-2, MOF@Ag-4, and MOF@Ag-6. The first weight loss step appears at the initial temperature (50 ${ }^{\circ} \mathrm{C}$ ), which is associated with the loss of guest molecules adsorbed physically on the materials and the values are fluctuant according to the initial solvation degree of the samples. The second weight loss step appears at about $320^{\circ} \mathrm{C}$, which is associated with the host weight loss, thermal decomposition of the MOF. The loading percentages of the MOF on Ag nanowires were calculated to be 28,49 , and $64 \%$ for MOF@Ag-2,MOF@Ag-4, and MOF@Ag-6, respectively, according to the inductively coupled plasma determination, via the formula of

Fe wt \% in the composites

Fe wt $\%$ in the MOF

The photocatalytic activity of MOF@Ag-2, MOF@Ag-4, and MOF@Ag-6 was tested on the degradation of MB. As illustrated in Figure 4a, 30 min of dark adsorption enabled MB to reach the adsorption equilibrium. In the process of dark adsorption, the adsorption capacity of different samples was different. The adsorption capacity of pure silver and MOFs was the minimum and the maximum, respectively. While the adsorption capacities of the hybrid materials were in the middle, among which the adsorption capacity increased with the increase of the loading percentage of the MOF on $\mathrm{Ag}$ nanowires, which is in accordance with the previous report. ${ }^{52}$ However, the situation was quite different when the light was turned on.MOF@Ag-6 achieved a near complete degradation first, spending about $50 \mathrm{~min} . \mathrm{MOF} @ \mathrm{Ag}-4$ was a close second, spending about $55 \mathrm{~min}$ to achieve a near complete degradation. Interestingly, the photocatalytic activity of MOF@Ag-2 had fallen behind that of pure MOFs despite being ahead of that of pure Ag nanowires. The difference of catalytic activity was more obvious in the kinetics study of the photocatalytic reaction (Figure $4 \mathrm{~b}$ ). Based on previous studies, ${ }^{53}$ the degradation of dyes can be attributed to the pseudo-first-order reaction with a simplified LangmuirHinshelwood model when $C_{0}$ is very small: $\ln \left(C_{0} / C_{t}\right)=k t$, where $k$ is the apparent first-order rate constant. Therefore, the constants $k$ are calculated by the slope of the plots as 0.04 , 0.14, 0.18,0.09, and 0.01 min ${ }^{-1}$ for MOF@Ag-2,MOF@Ag-4, MOF@Ag-6, pure MOFs, and pure Ag nanowires, respectively. It can be seen that $\mathrm{Ag}$ nanowires had a certain photocatalytic activity, although not very strong, which was considered to be from the plasmon photocatalysis. ${ }^{54}$ The recycle and repeated degradation performances for 5 runs' operation are provided in the Supporting Information (Figure S3), along with PXRD and SEM observation of the used MOF@Ag-6. No significant loss of the catalytic activity and crystallinity of MOF@Ag-6 was detected, indicating the stability of the hybrid catalyst.

Photoelectrochemical measurements were performed to investigate the activity difference among the catalysts. As illustrated in Figure 5a, UV-vis DRS spectra of MOF@Ag-2, MOF@Ag-4, MOF@Ag-6, and pure MOF are presented. All the catalysts presented a strong absorbance between 200 and $400 \mathrm{~nm}$, which was mainly associated with the ligand-to-metal charge transfer of $\mathrm{O}^{2-} \rightarrow \mathrm{Fe}^{3+}$ in MOFs. ${ }^{43} \mathrm{MOF} @ \mathrm{Ag}-2$, MOF@Ag-4, and MOF@Ag-6 showed decreased absorbance between 200 and $550 \mathrm{~nm}$, while increased absorbance between 550 and $800 \mathrm{~nm}$, compared with that of the pure MOF. The enhanced part of the absorbance in MOF@Ag-2, MOF@Ag-4, and MOF@Ag-6 comes mainly from the SPR-enhanced visible-near-infrared-driven excitation of $\mathrm{Ag}$ nanowires. ${ }^{55,56}$ The photocurrent transient response of each photocatalyst was displayed in Figure 5b, all hybrid catalysts including MOF@ Ag-2, MOF@Ag-4, and MOF@Ag-6 displayed enhanced photocurrent density compared with that of pure MOFs, suggesting a more efficient separation of the photogenerated electron-hole pairs, which can be further proved by the PL results. From the observation of Figure 5c, a strong band at $620-660 \mathrm{~nm}$ with a peak at $645 \mathrm{~nm}$ was detected in the pure MOF under an excitation wavelength of $320 \mathrm{~nm}$. In addition, it can be observed that the intensity of the PL emission followed the order pure MOF > MOF@Ag-2 > MOF@Ag-4 > MOF@ Ag-6. A lower PL intensity is generally indicative of a lower recombination rate of photogenerated charge carriers. Thus, we can conclude that the charge-separation efficiency follows the order MOF@Ag-6 > MOF@Ag-4 > MOF@Ag-2 > pure MOF. It could be concluded that the intimate interfacial contact between the two components in the hybrid catalyst facilitates the separation of photogenerated electrons and holes. However, the electron-hole separation efficiency order is not completely consistent with that of the results of $\mathrm{MB}$ degradation which followed MOF@Ag-6 > MOF@Ag-4 > pure MOF > MOF@Ag-2. It is considered that the BrunauerEmmett-Teller (BET) surface area of the catalyst also affects the catalytic efficiency through influencing the transmission and contact probability of reactants. The BET surface areas of MOF@Ag-2,MOF@Ag-4, MOF@Ag-6, and pure MOF are about 146, 342, 437, and $1028 \mathrm{~m}^{2} \mathrm{~g}^{-1}$ (Figure S2b), respectively, following the order of pure MOF > MOF@Ag$6>$ MOF@Ag-4 > MOF@Ag-2. Combining the two factors of electron-hole separation efficiency and BET surface area, the 

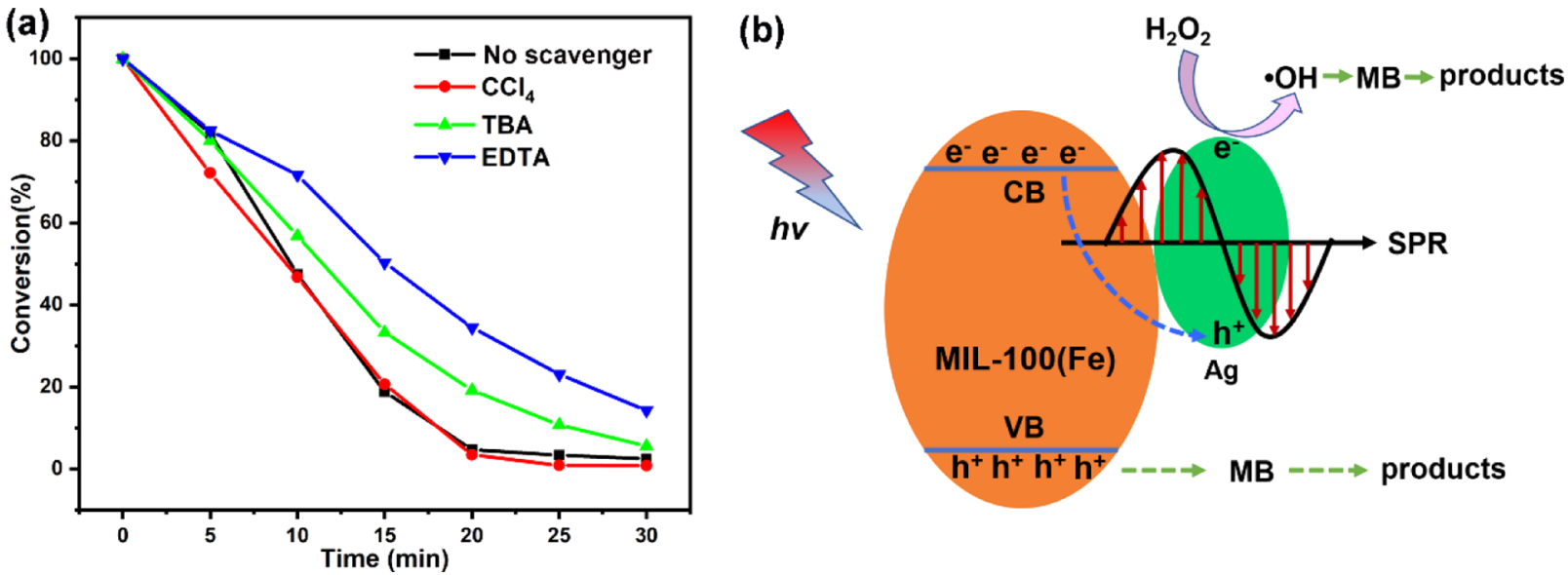

Figure 6. Influences of scavengers on the conversion of $\mathrm{MB}(\mathrm{a})$ and illustration of the proposed mechanism of MB degradation over the MOF layer covering the Ag nanowire hybrid catalyst (b).

photocatalytic activity order follows MOF@Ag-6 > MOF@Ag4 > pure MOF > MOF@Ag-2.

The active species trapping experiments were conducted over MOF@Ag-6, where disodiumethylenediamine tetraacetate (EDTA), $\mathrm{CCl}_{4}$, and tert-butylalcohol (TBA) were used as a scavenger for the hole, electron, and hydroxyl radical $\left({ }^{\bullet} \mathrm{OH}\right)$, respectively. As illustrated in Figure 6a, the addition of EDTA and TBA can induce an obvious decrease in photocatalytic activity of MOF@Ag-6, while $\mathrm{CCl}_{4}$ has no obvious shield effect on the photodegradation of $\mathrm{MB}$. This result confirms that holes and ${ }^{\circ} \mathrm{OH}$ are the main radical species in the $\mathrm{MB}$ photodegradation process. On account of the results above, a reaction mechanism for photocatalytic degradation of $\mathrm{MB}$ over the hybrid catalyst of the MOF coating layer on $\mathrm{Ag}$ nanowire is proposed (Figure $6 \mathrm{~b}$ ). Under irradiation, the electrons exited from the valence band of MOFs to the conduction band generating electrons and holes. Subsequently, the Ag nanowire, which is SPR-excited, can accept the photogenerated electrons and the lifetime of charge carriers are prolonged. The SPRexcited electron of the $\mathrm{Ag}$ nanowire would be transported from Ag to $\mathrm{H}_{2} \mathrm{O}_{2}$ forming ${ }^{\circ} \mathrm{OH}$ to complete the oxidation of MB. In addition, the photogenerated holes, which generally contribute to the oxidation reaction, of the MOF can directly oxidize the adsorbed MB.

\section{CONCLUSIONS}

A MOF layer was controllably coated on the surface of $\mathrm{Ag}$ nanowires to investigate the photocatalytic performance of the $\mathrm{MOF}-\mathrm{Ag}$ composite nanowire on the degradation of organic dyes. The hybrid catalyst of MOF-Ag nanowires showed enhanced photogenerated electron-hole separation efficiency compared with that of the pure MOF. However, the BET surface area of the hybrid catalyst was lower than that of the pure MOF. The photocatalytic activity was influenced by both the photogenerated electron-hole separation efficiency and the BET surface area. Therefore, the hybrid catalyst with a thicker MOF coating layer has more advantages in the photocatalytic degradation of $\mathrm{MB}$, suggesting the importance of the controlling of the MOF coating layer on the SPR-excited Ag nanowire.

\section{EXPERIMENTAL SECTION}

The preparation of Ag nanowires and controllable coating Ag nanowires with the MOF layer were according to our previous work with minor modification. ${ }^{57}$

4.1. Preparation of Ag Nanowires. $0.2 \mathrm{~g}$ of polyvinylpyrrolidone was dissolved into $25 \mathrm{~mL}$ of ethylene glycol forming homogeneous solution, into which $3.5 \mathrm{~g}$ of $\mathrm{FeCl}_{3}$ ethylene glycol solution $(0.6 \mathrm{mM})$ and $0.25 \mathrm{~g}$ of $\mathrm{AgNO}_{3}$ solid were added. The mixture was stirred vigorously for $10 \mathrm{~min}$ until the solid was dissolved completely. The obtained solution was then transferred to a Teflon-lined stainless-steel autoclave and sealed to heat at $130{ }^{\circ} \mathrm{C}$. After reaction for $5 \mathrm{~h}$, the autoclave was cooled to room temperature. The obtained products were washed with acetone and ethanol for several times and finally dispersed in $20 \mathrm{~mL}$ of ethanol.

4.2. Controllable Coating Ag Nanowires with the MOF Layer. The above obtained Ag nanowire ethanol dispersion liquid was ultrasound dispersed evenly, and $5 \mathrm{~mL}$ of the uniform dispersion was added into the mixture of $5 \mathrm{~mL}$ of $\mathrm{FeCl}_{2} \cdot 6 \mathrm{H}_{2} \mathrm{O}$ aqueous solution $(8 \mathrm{mM})$ and $5 \mathrm{~mL}$ of mixture aqueous solution ( $8 \mathrm{mM}$ trimesic acid and $24 \mathrm{mM}$ of $\mathrm{NaOH}$ ). The mixture was kept under room temperature for $30 \mathrm{~min}$ for one cycle of MOF layer coating on Ag nanowires. To control the thickness of the MOF coating layer, several other MOF layer coating cycles on $\mathrm{Ag}$ nanowires were conducted by the similar procedure.

4.3. Evaluation of Photocatalytic Activity. The photocatalytic activity of the products was tested by the degradation of $\mathrm{MB}$ in aqueous solution under ultraviolet visible light irradiation by using a $500 \mathrm{~W}$ Xe arc lamp with a 300-780 nm light source. Typically, $5 \mathrm{mg}$ of the photocatalyst sample was added into $100 \mathrm{~mL}$ of $20 \mathrm{mg} / \mathrm{L} \mathrm{MB}$ aqueous solution. The suspension was magnetically stirred in the darkroom for 30 min to achieve adsorption equilibrium. After that, $0.5 \mathrm{~mL}$ of hydrogen peroxide solution (30\%) was added and the light was turned on. $2 \mathrm{~mL}$ of samples was taken out every $5 \mathrm{~min}$ and was centrifuged for the supernatant. The concentration of MB left in the supernatant solution was determined by using a UV$4802 S$ UV-visible spectrophotometer at its maximum absorption wavelength of $664 \mathrm{~nm}$. 


\section{ASSOCIATED CONTENT}

\section{SI Supporting Information}

The Supporting Information is available free of charge at https://pubs.acs.org/doi/10.1021/acsomega.0c05229.

Materials and instrumentation; SEM imaging of $\mathrm{Ag}$ nanowires; FT-IR and $\mathrm{N}_{2}$ adsorption-desorption curves; and stability test of MOF@Ag-6 (PDF)

\section{AUTHOR INFORMATION}

\section{Corresponding Authors}

Xi Chen - School of Materials Science and Engineering, East China Jiaotong University, Nanchang 330013, People's Republic of China; (1) orcid.org/0000-0001-7655-3910; Email: chenxi731@foxmail.com

Kun Yao - Shenzhen Zhongxing New Material Technology Company Ltd., Shenzhen 518000, People's Republic of China; Email: 381003700@qq.com

Yongxin Liu - School of Materials Science and Engineering, East China Jiaotong University, Nanchang 330013, People's Republic of China; 이이.org/0000-0002-3665-2209; Email: liuyx629@foxmail.com

\section{Authors}

Yanshuang Zhang - School of Materials Science and Engineering, East China Jiaotong University, Nanchang 330013, People's Republic of China

Xiangyun Kong - School of Materials Science and Engineering, East China Jiaotong University, Nanchang 330013, People's Republic of China

Lingzhi Liu - State Key Laboratory of Polymer Physics and Chemistry, Changchun Institute of Applied Chemistry, Chinese Academy of Sciences, Changchun 130022, People's Republic of China

Jiali Zhang - School of Materials Science and Engineering, East China Jiaotong University, Nanchang 330013, People's Republic of China; 10 orcid.org/0000-0002-3268-9450

Zanru Guo - School of Materials Science and Engineering, East China Jiaotong University, Nanchang 330013, People's Republic of China; (1) orcid.org/0000-0003-1091-1409

Wenyuan Xu - School of Materials Science and Engineering, East China Jiaotong University, Nanchang 330013, People's Republic of China; 10 orcid.org/0000-0002-4519-0413

Zhili Fang - School of Materials Science and Engineering, East China Jiaotong University, Nanchang 330013, People's Republic of China

Complete contact information is available at:

https://pubs.acs.org/10.1021/acsomega.0c05229

\section{Notes}

The authors declare no competing financial interest.

\section{ACKNOWLEDGMENTS}

This work is supported by the Youth Science Fund through the Science and Technology Project of Jiangxi Province (20192BAB216014 and 20202BABL213023), the Science and Technology Research Projects of Jiangxi Province Education Department (GJJ180346), and National Natural Science Foundation of China (21865010, 21865009, and 21872049).

\section{REFERENCES}

(1) Crini, G. Non-conventional low-cost adsorbents for dye removal: A review. Bioresour. Technol. 2006, 97, 1061-1085.

(2) Peng, X.; Huang, D.; Odoom-Wubah, T.; Fu, D.; Huang, J.; Qin, Q. Adsorption of anionic and cationic dyes on ferromagnetic ordered mesoporous carbon from aqueous solution: Equilibrium, thermodynamic and kinetics. J. Colloid Interface Sci. 2014, 430, 272-282.

(3) Katheresan, V.; Kansedo, J.; Lau, S. Y. Efficiency of various recent wastewater dye removal methods: A review. J. Environ. Chem. Eng. 2018, 6, 4676-4697.

(4) Xu, W.; Qin, X.; Kuang, X. Study on Catalytic Treatment of Simulated Wastewater by $\mathrm{TiO}_{2} / \mathrm{RGO}$ and $\mathrm{Fe}_{3} \mathrm{O}_{4} / \mathrm{RGO}$. J. East China Jiaot. Univ. 2019, 36, 109-114.

(5) Shen, L.; Liang, R.; Wu, L. Strategies for engineering metalorganic frameworks as efficient photocatalysts. Chinese J. Catal. 2015, 36, 2071-2088.

(6) Wang, C.-C.; Li, J.-R.; Lv, X.-L.; Zhang, Y.-Q.; Guo, G. Photocatalytic organic pollutants degradation in metal-organic frameworks. Energy Environ. Sci. 2014, 7, 2831-2867.

(7) Natarajan, S.; Bajaj, H. C.; Tayade, R. J. Recent advances based on the synergetic effect of adsorption for removal of dyes from waste water using photocatalytic process. J. Environ. Sci. 2018, 65, 201-222.

(8) Daghrir, R.; Drogui, P.; Robert, D. Modified $\mathrm{TiO}_{2}$ For Environmental Photocatalytic Applications: A Review. Ind. Eng. Chem. Res. 2013, 52, 3581-3599.

(9) Tripathy, N.; Ahmad, R.; Kuk, H.; Lee, D. H.; Hahn, Y.-B.; Khang, G. Rapid methyl orange degradation using porous $\mathrm{ZnO}$ spheres photocatalyst. J. Photochem. Photobiol., B 2016, 161, 312-317.

(10) Ebrahimi, R.; Maleki, A.; Zandsalimi, Y.; Ghanbari, R.; Shahmoradi, B.; Rezaee, R.; Safari, M.; Joo, S. W.; Daraei, H.; Harikaranahalli Puttaiah, S.; Giahi, O. Photocatalytic degradation of organic dyes using $\mathrm{WO}_{3}$-doped $\mathrm{ZnO}$ nanoparticles fixed on a glass surface in aqueous solution. J. Ind. Eng. Chem. 2019, 73, 297-305.

(11) Cheng, L.; Xiang, Q.; Liao, Y.; Zhang, H. CdS-Based photocatalysts. Energy Environ. Sci. 2018, 11, 1362-1391.

(12) Ye, Z.; Kong, L.; Chen, F.; Chen, Z.; Lin, Y.; Liu, C. A comparative study of photocatalytic activity of $\mathrm{ZnS}$ photocatalyst for degradation of various dyes. Optik 2018, 164, 345-354.

(13) Murray, L. J.; Dincă, M.; Long, J. R. Hydrogen storage in metal-organic frameworks. Chem. Soc. Rev. 2009, 38, 1294-1314.

(14) Saha, D.; Bao, Z.; Jia, F.; Deng, S. Adsorption of $\mathrm{CO}_{2}, \mathrm{CH}_{4}$ $\mathrm{N}_{2} \mathrm{O}$, and $\mathrm{N}_{2}$ on MOF-5, MOF-177, and Zeolite 5A. Environ. Sci. Technol. 2010, 44, 1820-1826.

(15) Banerjee, R.; Phan, A.; Wang, B.; Knobler, C.; Furukawa, H.; O'Keeffe, M.; Yaghi, O. M. High-Throughput Synthesis of Zeolitic Imidazolate Frameworks and Application to $\mathrm{CO}_{2}$ Capture. Science 2008, 319, 939

(16) Xu, G.; Zhang, X.; Guo, P.; Pan, C.; Zhang, H.; Wang, C. Mn ${ }^{\mathrm{II}}$ based MIL-53 Analogues: Synthesis Using Neutral Bridging $\mu_{2-}$ Ligands and Application in Liquid-Phase Adsorption and Separation of C6-C8 Aromatics. J. Am. Chem. Soc. 2010, 132, 3656-3657.

(17) Li, J.-R.; Sculley, J.; Zhou, H.-C. Metal-Organic Frameworks for Separations. Chem. Rev. 2012, 112, 869-932.

(18) Li, J.-R.; Kuppler, R. J.; Zhou, H.-C. Selective gas adsorption and separation in metal-organic frameworks. Chem. Soc. Rev. 2009, 38, 1477-1504.

(19) Lee, J.; Farha, O. K.; Roberts, J.; Scheidt, K. A.; Nguyen, S. T.; Hupp, J. T. Metal-organic framework materials as catalysts. Chem. Soc. Rev. 2009, 38, 1450-1459.

(20) Corma, A.; García, H.; Llabrés i Xamena, F. X. Engineering Metal Organic Frameworks for Heterogeneous Catalysis. Chem. Rev. 2010, 110, 4606-4655.

(21) Zhu, L.; Liu, X.-Q.; Jiang, H.-L.; Sun, L.-B. Metal-Organic Frameworks for Heterogeneous Basic Catalysis. Chem. Rev. 2017, 117, $8129-8176$

(22) Horcajada, P.; Serre, C.; Vallet-Regí, M.; Sebban, M.; Taulelle, F.; Férey, G. Metal-Organic Frameworks as Efficient Materials for Drug Delivery. Angew. Chem., Int. Ed. 2006, 45, 5974-5978. 
(23) Khaletskaya, K.; Reboul, J.; Meilikhov, M.; Nakahama, M.; Diring, S.; Tsujimoto, M.; Isoda, S.; Kim, F.; Kamei, K.-i.; Fischer, R. A.; Kitagawa, S.; Furukawa, S. Integration of Porous Coordination Polymers and Gold Nanorods into Core-Shell Mesoscopic Composites toward Light-Induced Molecular Release. J. Am. Chem. Soc. 2013, 135, 10998-11005.

(24) Taylor-Pashow, K. M. L.; Della Rocca, J.; Xie, Z.; Tran, S.; Lin, W. Postsynthetic Modifications of Iron-Carboxylate Nanoscale Metal-Organic Frameworks for Imaging and Drug Delivery. J. Am. Chem. Soc. 2009, 131, 14261-14263.

(25) Kreno, L. E.; Leong, K.; Farha, O. K.; Allendorf, M.; Van Duyne, R. P.; Hupp, J. T. Metal-Organic Framework Materials as Chemical Sensors. Chem. Rev. 2012, 112, 1105-1125.

(26) Lu, G.; Hupp, J. T. Metal-Organic Frameworks as Sensors: A ZIF-8 Based Fabry-Pérot Device as a Selective Sensor for Chemical Vapors and Gases. J. Am. Chem. Soc. 2010, 132, 7832-7833.

(27) Liu, L.; Zhou, Y.; Liu, S.; Xu, M. The Applications of MetalOrganic Frameworks in Electrochemical Sensors. ChemElectroChem 2018, 5, 6-19.

(28) Xiao, J.-D.; Jiang, H.-L. Metal-Organic Frameworks for Photocatalysis and Photothermal Catalysis. Acc. Chem. Res. 2019, 52, 356-366.

(29) Wen, L.-L.; Wang, F.; Feng, J.; Lv, K.-L.; Wang, C.-G.; Li, D.-F. Structures, Photoluminescence, and Photocatalytic Properties of Six New Metal-Organic Frameworks Based on Aromatic Polycarboxylate Acids and Rigid Imidazole-Based Synthons. Cryst. Growth Des. 2009, 9, 3581-3589.

(30) Das, M. C.; Xu, H.; Wang, Z.; Srinivas, G.; Zhou, W.; Yue, Y.F.; Nesterov, V. N.; Qian, G.; Chen, B. A $\mathrm{Zn}_{4} \mathrm{O}$-containing doubly interpenetrated porous metal-organic framework for photocatalytic decomposition of methyl orange. Chem. Commun. 2011, 47, 1171511717.

(31) Llabrés i Xamena, F. X.; Corma, A.; Garcia, H. Applications for Metal-Organic Frameworks (MOFs) as Quantum Dot Semiconductors. J. Phys. Chem. C 2007, 111, 80-85.

(32) Shen, L.; Wu, W.; Liang, R.; Lin, R.; Wu, L. Highly dispersed palladium nanoparticles anchored on $\mathrm{UiO}-66\left(\mathrm{NH}_{2}\right)$ metal-organic framework as a reusable and dual functional visible-light-driven photocatalyst. Nanoscale 2013, 5, 9374-9382.

(33) Laurier, K. G. M.; Vermoortele, F.; Ameloot, R.; De Vos, D. E.; Hofkens, J.; Roeffaers, M. B. J. J. Iron(III)-Based Metal-Organic Frameworks As Visible Light Photocatalysts. J. Am. Chem. Soc. 2013, 135, 14488-14491.

(34) Jing, H.-P.; Wang, C.-C.; Zhang, Y.-W.; Wang, P.; Li, R. Photocatalytic degradation of methylene blue in ZIF-8. RSC Adv. 2014, 4, 54454-54462.

(35) Jiang, D.; Xu, P.; Wang, H.; Zeng, G.; Huang, D.; Chen, M.; Lai, C.; Zhang, C.; Wan, J.; Xue, W. Strategies to improve metal organic frameworks photocatalyst's performance for degradation of organic pollutants. Coord. Chem. Rev. 2018, 376, 449-466.

(36) Younis, S. A.; Kwon, E. E.; Qasim, M.; Kim, K.-H.; Kim, T.; Kukkar, D.; Dou, X.; Ali, I. Metal-organic framework as a photocatalyst: Progress in modulation strategies and environmental/ energy applications. Prog. Energy Combust. Sci. 2020, 81, 100870.

(37) Zhang, X.; Yang, Y.; Huang, W.; Yang, Y.; Wang, Y.; He, C.; Liu, N.; Wu, M.; Tang, L. g- $\mathrm{C}_{3} \mathrm{~N}_{4} / \mathrm{UiO}-66$ nanohybrids with enhanced photocatalytic activities for the oxidation of dye under visible light irradiation. Mater. Res. Bull. 2018, 99, 349-358.

(38) Liang, Q.; Cui, S.; Liu, C.; Xu, S.; Yao, C.; Li, Z. Construction of CdS@UIO-66- $\mathrm{NH}_{2}$ core-shell nanorods for enhanced photocatalytic activity with excellent photostability. J. Colloid Interface Sci. 2018, 524, 379-387.

(39) Su, Z.; Zhang, B.; Shi, J.; Tan, D.; Zhang, F.; Liu, L.; Tan, X.; Shao, D.; Yang, G.; Zhang, J. An $\mathrm{NH}_{2}-\mathrm{MIL}-125(\mathrm{Ti}) / \mathrm{Pt} / \mathrm{g}-\mathrm{C}_{3} \mathrm{~N}_{4}$ catalyst promoting visible-light photocatalytic $\mathrm{H} 2$ production. Sustainable Energy Fuels 2019, 3, 1233-1238.

(40) Wang, S. S.; Jiao, L.; Qian, Y.; Hu, W. C.; Xu, G. Y.; Wang, C.; Jiang, H. L. Boosting Electrocatalytic Hydrogen Evolution over
Metal-Organic Frameworks by Plasmon-Induced Hot-Electron Injection. Angew. Chem., Int. Ed. 2019, 58, 10713-10717.

(41) Li, S.; Miao, P.; Zhang, Y.; Wu, J.; Zhang, B.; Du, Y.; Han, X.; Sun, J.; Xu, P. Recent Advances in Plasmonic Nanostructures for Enhanced Photocatalysis and Electrocatalysis. Adv. Mater. 2020, 2000086.

(42) Wang, M.; Tang, Y.; Jin, Y. Modulating Catalytic Performance of Metal-Organic Framework Composites by Localized Surface Plasmon Resonance. ACS Catal. 2019, 9, 11502-11514.

(43) Liang, R.; Jing, F.; Shen, L.; Qin, N.; Wu, L. M@MIL-100(Fe) $(\mathrm{M}=\mathrm{Au}, \mathrm{Pd}, \mathrm{Pt})$ nanocomposites fabricated by a facile photodeposition process: Efficient visible-light photocatalysts for redox reactions in water. Nano Res. 2015, 8, 3237-3249.

(44) Awazu, K.; Fujimaki, M.; Rockstuhl, C.; Tominaga, J.; Murakami, H.; Ohki, Y.; Yoshida, N.; Watanabe, T. A Plasmonic Photocatalyst Consisting of Silver Nanoparticles Embedded in Titanium Dioxide. J. Am. Chem. Soc. 2008, 130, 1676-1680.

(45) Jing, Y.; Lei, Q.; Xia, C.; Guan, Y.; Yang, Y.; He, J.; Yang, Y.; Zhang, Y.; Yan, M. Synthesis of $\mathrm{Ag}$ and $\mathrm{AgCl}$ co-doped ZIF-8 hybrid photocatalysts with enhanced photocatalytic activity through a synergistic effect. RSC Adv. 2020, 10, 698-704.

(46) Duan, C.; Liu, C.; Meng, X.; Gao, K.; Lu, W.; Zhang, Y.; Dai, L.; Zhao, W.; Xiong, C.; Wang, W.; Liu, Y.; Ni, Y. Facile synthesis of Ag NPs@ MIL-100(Fe)/ guar gum hybrid hydrogel as a versatile photocatalyst for wastewater remediation: Photocatalytic degradation, water/oil separation and bacterial inactivation. Carbohyd. Polym. 2020, 230, 115642 .

(47) Liu, Y.; Xie, Y.; Dai, M.; Gong, Q.; Dang, Z. Ag/AgCl/MIL101 $(\mathrm{Fe})$ Catalyzed Degradation of Methylene Blue under Visible Light Irradation. Materials 2019, 12, 1453.

(48) Wiley, B. J.; Im, S. H.; Li, Z.-Y.; McLellan, J.; Siekkinen, A.; Xia, Y. Maneuvering the Surface Plasmon Resonance of Silver Nanostructures through Shape-Controlled Synthesis. J. Phys. Chem. B 2006, 110, $15666-15675$.

(49) Horcajada, P.; Surblé, S.; Serre, C.; Hong, D.-Y.; Seo, Y.-K.; Chang, J.-S.; Grenèche, J.-M.; Margiolaki, I.; Férey, G. Synthesis and catalytic properties of MIL-100(Fe), an iron(iii) carboxylate with large pores. Chem. Commun. 2007, 2820-2822.

(50) Guesh, K.; Caiuby, C. A. D.; Mayoral, Á.; Díaz-García, M.; Díaz, I.; Sanchez-Sanchez, M. Sustainable Preparation of MIL$100(\mathrm{Fe})$ and Its Photocatalytic Behavior in the Degradation of Methyl Orange in Water. Cryst. Growth Des. 2017, 17, 1806-1813.

(51) Chen, D.-D.; Yi, X.-H.; Zhao, C.; Fu, H.; Wang, P.; Wang, C.C. Polyaniline modified MIL-100(Fe) for enhanced photocatalytic $\mathrm{Cr}(\mathrm{VI})$ reduction and tetracycline degradation under white light. Chemosphere 2020, 245, 125659.

(52) Liu, Y.; Wang, S.; Lu, Y.; Zhao, Y.; Zhang, Y.; Xu, G.; Zhang, J.; Fang, Z.; Xu, W.; Chen, X. Loading Control of Metal-Organic Frameworks in $\mathrm{Fe}_{3} \mathrm{O}_{4} @ \mathrm{MOF}$ Series Composite Adsorbents for Optimizing Dye Adsorption. Ind. Eng. Chem. Res. 2019, 58, 2224422249.

(53) Liang, R.; Jing, F.; Shen, L.; Qin, N.; Wu, L. MIL-53(Fe) as a highly efficient bifunctional photocatalyst for the simultaneous reduction of $\mathrm{Cr}(\mathrm{VI})$ and oxidation of dyes. J. Hazard. Mater. 2015, $287,364-372$.

(54) Linic, S.; Christopher, P.; Ingram, D. B. Plasmonic-metal nanostructures for efficient conversion of solar to chemical energy. Nat. Mater. 2011, 10, 911-921.

(55) Qiao, P.; Sun, B.; Li, H.; Pan, K.; Tian, G.; Wang, L.; Zhou, W. Surface Plasmon Resonance-Enhanced Visible-NIR-Driven Photocatalytic and Photothermal Catalytic Performance by $\mathrm{Ag} / \mathrm{Mesoporous}$ Black TiO2 Nanotube Heterojunctions. Chem.-Asian J. 2019, 14, $177-186$

(56) Liu, X.; He, L.; Zheng, J.; Guo, J.; Bi, F.; Ma, X.; Zhao, K.; Liu, Y.; Song, R.; Tang, Z. Solar-Light-Driven Renewable Butanol Separation by Core-Shell Ag@ZIF-8 Nanowires. Adv. Mater. 2015, 27, 3273-3277.

(57) Chen, X.; Zhang, Y.; Kong, X.; Guo, Z.; Xu, W.; Fang, Z.; Wang, S.; Liu, L.; Liu, Y.; Zhang, J. Controlling crystal growth of MIL- 
$100(\mathrm{Fe})$ on $\mathrm{Ag}$ nanowire surface for optimizing catalytic performance.

RSC Adv. 2020, 10, 25260-25265. 